\title{
SPONTANEOUS DISCONTINUITIES IN GALACTIC MAGNETIC FIELDS AND THE CREATION OF GALACTIC X-RAY HALOS
}

\author{
E.N. Parker \\ Laboratory for Astrophysics \\ University of Chicago \\ 933 East 56th Street, Chicago, Illinois 60637, USA
}

\begin{abstract}
The magnetic field in the gaseous disk of the galaxy is dynamically unstable to undulations with wavelengths of the order of $1 \mathrm{kpc}$. The elevated portions of the field are subject to rapid inflation $(\sim 50 \mathrm{~km} / \mathrm{sec})$ by the cosmic rays produced within the gaseous disk. The result is a magnetic halo of $1-3 \times 10^{-6}$ gauss, composed of close packed bipolar lobes of field extending outward from the surface of the disk to distances of several kpc. The inflation is presumably irregular, producing tangential discontinuities (current sheets) throughout the extended bipolar fields. A major portion of the magnetic energy is dissipated by rapid reconnection at these current sheets, heating the tenuous halo gas to temperatures of $10^{6}-10^{7}{ }^{\circ} \mathrm{K}$ and producing the $\mathrm{X}$-ray emission observed from the halos of many spiral galaxies.
\end{abstract}

It has been known for some time that spiral galaxies possess extended radio halos. In particular, it has been shown that many edge-on spiral galaxies have X-ray emitting halos or coronas (Forman, Jones and Tucker, 1985; Fabbiano and Trinchieri, 1984; Fabbiano, 1988) with total X-ray luminosities of $10^{39}-10^{42} \mathrm{ergs} / \mathrm{sec}$. Note, too, that some have only weak emission (Bregman and Glassgold, 1982), if any at all, with X-ray luminosities below $10^{39} \mathrm{ergs} / \mathrm{sec}$. One infers a similar distribution of halo $\mathrm{X}$-ray luminosities among spiral galaxies that are not edge-on to the line of sight. The observed X-ray emission implies halo gas densities of the order of $10^{-3} / \mathrm{cm}^{3}$ at $10^{6}-10^{7} \mathrm{~K}$.

The X-ray emission is sufficiently intense that the cooling times of the galactic halo are small compared to the Hubble time, raising the question of the heat source that maintains the temperature of the emitting gas. It is the purpose of this communication to suggest that the principal source of heat to the X-ray emitting halo gas comes from the cosmic rays generated in the gaseous disk of the galaxy. The energy is transferred from the cosmic rays into the halo magnetic fields and then channeled into the gas via dissipation at the tangential discontinuities throughout the magnetic field of the halo. 
Magnetic fields in the halo of our galaxy are evidently of the general order of $3 \times 10^{-6}$ gauss, based on the evidence that the cosmic rays, (observed at Earth to have a pressure of about $0.7 \times 10^{-12}$ dynes $/ \mathrm{cm}^{2}$ ) circulate freely between the gaseous disk of the galaxy and the halo. We assume the same field strength to be characteristic of galaxies known to possess X-ray halos.

The first question to be addressed is why a galaxy possesses an extended halo. The development of a halo begins with the fact (Parker, 1966, 1968, 1969) that the magnetic field within the gaseous disk of the galaxy is subject to a strong largescale instability, on wavelengths along the field of the order of a kpc. The instability involves the interaction of the interstellar gas and the cosmic ray gas through the magnetic field to which both gases are tightly coupled. The result of the instability is to push the interstellar gas into cloud complexes, which anchor the magnetic field. The field bulges upward out of the disk in the low density regions between the clouds, forming bipolar lobes of field (see simulations in Shibata, et al 1989).

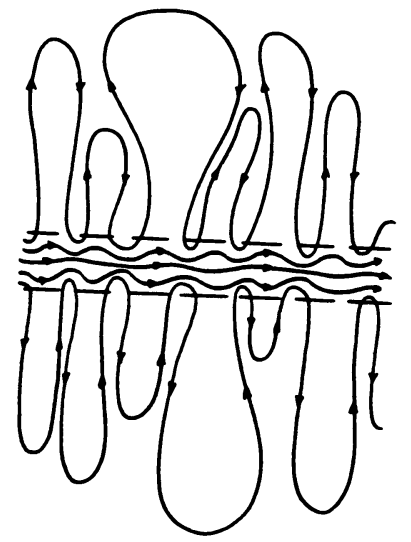

FIG. 1: Sketch of the bipolar lobes of magnetic field extending through the surfaces (dashed lines) of the gaseous disk of the galaxy.

Now cosmic rays are generated in the gaseous disk (presumably by supernovae) at a rate of the general order of $3 \times 10^{40} \mathrm{ergs} / \mathrm{sec}$, based on a cosmic ray life of about $2 \times 10^{6}$ years in the disk (deduced from a penetration through $5-6 \mathrm{gm} / \mathrm{cm}^{2}$ in a mean density of about $2 \mathrm{H}$ atoms $/ \mathrm{cm}^{3}$ ) and a mean energy density of $U \cong 2 \times 10^{-12}$ ergs $/ \mathrm{cm}^{3}$. The cosmic rays, which form a relativistic gas, are a powerful force in shaping the gaseous disk and the associated magnetic fields. The cosmic ray pressure $P \cong \frac{1}{3} U$ builds up until it inflates the bipolar lobes of field extending out 
from the disk. The inflation of the galactic magnetic field is the means by which the cosmic rays escape from the gaseous disk of the galaxy. The rate of inflation of the intercloud lobes of the galactic field is estimated at $30-60 \mathrm{~km} / \mathrm{sec}$, based on a half thickness $100 \mathrm{pc}$ for the disk and the cosmic ray dwell time of about $2 \times 10^{6}$ years in the disk. Hence we expect bipolar fields to sprout thickly from both surfaces of the gaseous disk and to extend far out from the disk as a consequence of their rapid inflation by the cosmic ray gas (Parker, 1965, 1968, 1969). Fig. 1 is a sketch of a vertical section through the gaseous disk, showing the extended lobes on either side. Recent observations (Hummel et al 1988, Beck, 1989) show the alignment of halo fields more or less perpendicular to the disk of the galaxy, providing observational support for the halo composed of close packed bipolar lobes. The magnetic energy of the extended lobes comes from the expanding cosmic rays, with the result that the rate at which the magnetic energy increases is a major fraction of the total cosmic ray production rate.

Presumably the lobes extend farther and farther, as they are inflated, until finally reconnection of the field cuts them loose from the galaxy. Reconnection proceeds only at a small fraction of the Alfven speed. Therefore, we expect to find that the bipolar magnetic lobes extend outward over distances many times larger than their kiloparsec widths. It is not possible to be more quantitative, because of the complicated and quantitatively intractable character of the dynamical reconnection. The statement is simply that cosmic rays seem to be a major effect in producing (inflating) the magnetic halo of a galaxy. The cosmic rays do work on the magnetic field at a rate equal to their own generation rate, estimated to be of the general order of the order of $3 \times 10^{40} \mathrm{ergs} / \mathrm{sec}$. Needless to say, there is also inflation by the gases from supernovae, producing chimneys etc. that undoubtedly contribute to the halo in some localities and there may be a galactic wind (see paper by H.J. Völk, these proceedings) which contributes to the halo phenomenon as well. The unique feature of the cosmic rays is their extreme mobility so that they inflate fields everywhere over the disk. So a galactic halo, with the magnetic lines of force largely perpendicular to the disk, appears to be an inevitable consequence of the activity, and particularly the cosmic ray production, in the disk (Parker, 1965, 1969).

Now the footpoints of the extended bipolar magnetic fields are sheared by the nonuniform rotation of the galaxy, and they are continuously intermixed by the motions of the interstellar gas, so that the lines of force within each lobe are wrapped and interwoven with characteristic scales that may be of the order of $10^{2} \mathrm{pc}$, based on the scale of the fluctuation $\Delta B$ of the field within the disk (Appenzeller, 1969, Jokipii and Lerche, 1969; Jokipii et al, 1969) and the general scale of interstellar clouds. What is more, the rapid inflation of the bipolar fields by the cosmic rays is not expected to be uniform, nor would the inflation of a bipolar field with complex 
internal field topology be uniform even if the applied cosmic ray pressure were uniform.

The essential point is that the irregular inflation and wrapping of the lines of force produces tangential discontinuities (current sheets) throughout the extended loops as an intrinsic part of the quasi-static equilibrium of the loops (Parker, 1972, 1979, $1982,1983 \mathrm{~b}, 1987,1989,1990)$. It should be noted too that tangential discontinuities arise where each bipolar loop presses against its neighbors and also between opposite legs of the individual bipolar loop. Indeed, it is these major current sheets, up the middle and between adjacent loops, that provide the reconnection of field lines that eventually frees the magnetic lobes and their cosmic rays from the galaxy. The discontinuities, being an intrinsic part of the equilibrium of the loop fields, are maintained throughout the dissipation of $\Delta B$ and $B$. The tangential discontinuities ultimately dissipate a major part of the magnetic energy of the extended bipolar loops of field, of which the galactic halo is composed. The magnetic energy was created by the expanding cosmic ray gas, so the final result is that a significant portion of the energy put into cosmic rays in the gaseous disk is ultimately channelled into heating the tenuous gases of the halo at a rate of the order of $3 \times 10^{40} \mathrm{ergs} / \mathrm{sec}$. It is interesting to note that Sturrock and Stein (1980) estimated earlier an energy input to the halo of our galaxy at about $3 \times 10^{39} \mathrm{ergs} / \mathrm{sec}$ as a result of differential rotation alone. On this basis, the heat input to the halo of our galaxy is somewhere between $10^{40}$ and $10^{41} \mathrm{ergs} / \mathrm{sec}$. It is interesting to note that this number falls in the middle of the range of the $\mathrm{X}$-ray luminosity of the halos of other galaxies.

If this conjecture on halo heating is correct, it follows that the X-ray luminosity of a galactic halo is an approximate measure of the general level of cosmic ray production in the galaxy. It would also follow that the galactic halo is a dynamical structure, similar in some ways to the X-ray corona of a main sequence star like the sun. The X-ray corona of the sun is created in the bipolar fields of active regions by the dissipation of the tangential discontinuities created by the equilibrium stress balance in the magnetic fields. The bipolar fields of the sun contain an irregular component $\Delta B(\sim B / 4)$ as a consequence of the random continuous mixing and swirling of the footpoints of the field at the photosphere. It is the dissipation of $\Delta B$ that is the principal source of heat for the $\mathrm{X}$-ray emitting gas (Parker, 1983b).

\section{REFERENCES}

Appenzeller, I. (1969), Astrophys. J. 151, 907-918.

Beck, R. (1989), IEEE Trans. Plasma Science (in press).

Bregman, J.N. and Glassgold, A.E. (1982), Astrophys. J. 263, 564-570.

Fabbiano, G. (1988), Astrophys. J. 330, 672-683.

Fabbiano, G. and Trinchieri, G. (1984), Astrophys. J. 286, 491-497. 
Forman, W., Jones, C., and Tucker, W. (1985), Astrophys. J. 293, 102-119.

Hummel, E., Lesch, H., Wielebinski, R., and Schlickeiser, R. (1988), Astron. Astrophys. 197, L29-L31.

Jokipii, J.R. and Lerche, I. (1969), Astrophys. J. 157, 1137-1145.

Jokipii, J.R., Lerche, I., and Schommer, R.A. (1969), Astrophys. J. Letters 157, L119-L124.

Parker, E.N. (1966), Astrophys. J. 145, 811-833.

Parker, E.N. (1965), Astrophys. J. 142, 584-590.

Parker, E.N. (1968), Chap. 14 in Stars and Stellar Systems, Vol. VII: Nebulae and Interstellar Matter, University of Chicago Press, Chicago. ed. B.M. Middlehurst and L.H. Allen.

Parker, E.N. (1969), Space Sci. Rev. 9, 651-672.

Parker, E.N. (1972), Astrophys. J. 174, 499-510.

Parker, E.N. (1979), Cosmical Magnetic Fields, Oxford University Press, Oxford.

Parker, E.N. (1982), Geophys. Astrophys. Fluid Dyn. 22, 195-218.

Parker, E.N. (1983a), Astrophys. J. 264, 642-647.

Parker, E.N. (1983b), Geophys. Astrophys. Fluid Dyn. 23, 85-102.

Parker, E.N. (1987), Astrophys. J. 318, 876-887.

Parker, E.N. (1989), Geophys. Astrophys. Fluid Dyn. (in press).

Parker, E.N. (1990), Geophys. Astrophys. Fluid Dyn. (in press).

Shibata, K., Tajima, T., Matsumoto, R., Horiuchi, T., Hanawa, T., Rosner, R., and

Uchida, Y. (1989), Astrophys. J. 338, 471-492.

Sturrock, P.A. and Stern, R. (1980), Astrophys. J. 238, 98-102.

BIERMANN: M82 has the most extensive X-ray halo known among disk galaxies. M82 also is well-known to have a high rate of star formation and supernova events. It does seem to fit your heating mechanism very well that the X-ray spectrum of M82 corresponds to such a hot gas that we could not even put a strong upper limit on the temperature with a best fit number of almost $10^{8} \mathrm{~K}$ (Schaaf et al., 1989, Astrophys. J. 336, 722).

KAHN: (1) The gas flow into a galactic fountain also inflates the magnetic field in the halo and produces a similar effect to that produced by the cosmic rays.

(2) The spectrum of the radiation produced in the gas by Joule heating will be characterized by radiation corresponding to all temperatures between the highest temperatures reached (near the current sheet) and zero $\mathrm{K}$.

DOGIEL: Current sheets are very effective for fast particle acceleration in the solar corona. According to your estimate the total energy power of current sheets in the halos of galaxies is about $10^{41} \mathrm{erg} / \mathrm{s}$. We need just 
this energy power to produce the cosmic-ray luminosity of our Galaxy.

In this connection is it necessary for us to change our point of view on the cosmic-ray origin and to suggest that a significant part of the cosmic-ray flux is generated in the halo?

PARKER: I think not. My suggestion was that the cosmic rays produce the halo. I cannot then argue that the halo produces a major part of the cosmic rays without violating the first law of thermodynamics. There may, of course, be some modest local acceleration of particles in the reconnection at a tangential discontinuity. What energies the particles reach I do not know. But I doubt that the process is so efficient that it feeds particles back into the cosmic ray state in significant quantities.

SPANGLER: The configuration you show, representing the magnetic field drawn out by cosmic rays, might be conclusive to the generation of MHD waves by cosmic ray streaming. The subsequent linear and nonlinear dissipation of these waves would then serve to heat the galactic corona. Do you have any opinions on the relative importance of this heating mechanism and the one you have proposed?

PARKER: The dissipation of current sheets (tangential discontinuities) is a complicated chaotic process, often involving brief bursts of reconnection interspersed with periods of relative quiet. I am sure that waves, jets of fluid, turbulence, fast particle acceleration, etc. are produced in the reconnection process. These are all part of the process by which the decaying magnetic energy is degraded into heat. The waves are generally of high frequency so they dissipate effectively. Unfortunately there is no way at present to provide a general quantitative description of the dynamical dissipation at a current sheet.

KULSRUD: How do you think cosmic rays, which have a very large effective conductivity, would effect the phenomena of magnetic reconnection in the halo?

PARKER: I have thought about this interesting question, and I do not know the answer. The cosmic rays are extremely mobile, and the Alfven speed in the cosmic ray gas alone is a major fraction of c. The thermal gas is, of course, very much less mobile (at $10^{6}-10^{7} \mathrm{~K}$ ) and at $10^{-3}$ atoms $/ \mathrm{cm}^{3}$ produces an Alfvén speed of $10^{2} \mathrm{~km} / \mathrm{s}$ in fields of a few microgauss or so. It would be interesting to work out what really might happen. In my present remarks I have merely relied on the principle that the rate of reconnection does not depend strongly on the effective magnetic Reynolds number, etc.

KUNDT: Your estimate of the cosmic-ray power produced by the galaxy depends on its storage volume $V$. If the cosmic rays are produced in the molecular-cloud layer, the required power is $P=\varepsilon V / \tau=10^{-12+66-14.5}$ $\mathrm{erg} / \mathrm{s}^{1}=10^{39.5} \mathrm{erg} / \mathrm{s}^{1}$ (cf. 1983, Astrophys. Space Sci. 90, 59).

PARKER: The cosmic ray life in the gaseous disk of the galaxy is estimated at about $2.10^{6}$ years based on the penetration of cosmic rays 
through $5-6 \mathrm{~g} / \mathrm{cm}^{2}$ in an interstellar medium of $2 \mathrm{H}$ atoms $/ \mathrm{cm}^{3}$. If the volume of the gaseous disk is estimated from a half thickness of $10^{2} \mathrm{pc}$ and a radius of $10^{4} \mathrm{pc}$, the result is approximately $1.7 .10^{66} \mathrm{~cm}^{3}$. The cosmic ray energy density here at the sun is approximately $2.10^{-12}$ $\mathrm{erg} / \mathrm{cm}^{3}$. Applying this to the entire disk volume we obtain $3.10^{54}$ ergs in the cosmic rays in the disk. A lifetime of $2.10^{6}$ years requires a mean input rate of $7.10^{40} \mathrm{erg} / \mathrm{s}$ to maintain the cosmic rays in the disk. There is, of course, some considerable uncertainty in these numbers, based on the simplifying assumptions. But I know of no way to obtain a result much smaller than the number quoted.

The input rate can also be computed from the mean age of $10^{7}$ years of the cosmic ray particles (inferred from the decay of $\mathrm{Be}^{10}$ cosmic ray nuclei). Given that in $10^{7}$ years the cosmic rays have penetrated $5-6 \mathrm{~g} / \mathrm{cm}^{2}$, it follows that the mean density of matter along their path is $0.3 \mathrm{H}$ atoms $/ \mathrm{cm}^{3}$. Evidently this involves spending about $1 / 6$ of their time in the gaseous disk of the galaxy and about $5 / 6$ in the galactic halo where the ambient gas density is negligible. The volume occupied by the cosmic rays is, then, about 6 times the volume of the disk, or $10^{67} \mathrm{~cm}^{3}$. This volume must be replenished each $10^{7}$ years which requires an input of $7.10^{40} \mathrm{erg} / \mathrm{s}$. The essential point is that cosmic rays do not remain in the gaseous disk for more than $2.10^{6}$ years, for, if they did, they would penetrate through more than $5-6 \mathrm{~g} / \mathrm{cm}^{2}$. Hence the equivalent of the cosmic rays in the volume occupied by the gaseous disk must be replaced each $2 \cdot 10^{6}$ years.

DEINZER: What is your argument against van Ballegooijen who maintains that there are no discontinuities evolving, if they were not in the starting conditions?

PARKER: I know of no argument for van Ballegooijen. The general theory shows the existence of tangential discontinuities as an intrinsic part of the static equilibrium of all but the simplest field topologies, and many formal examples have now been constructed to illustrate the phenomenon. Van Ballegooijen choses to ignore these facts. 